\title{
Glial and axonal body fluid biomarkers are related to infarct volume, severity and outcome
}
A. Petzold MD PhD *
P. Michel MD †
M. Stock MD
M. Schluep $\operatorname{PhD} \S$

December 13, 2009

Keywords: stroke, biomarker, cerebrospinal fluid, serum

*Corresponding author: Department of Neuroimmunology, Institute of Neurology \& The Tavistock Intensive Care Unit, The National Hospital for Neurology and Neurosurgery, Queen Square, London, WC1N 3BG, United Kingdom. Fax: +44 207837 8553, E-mail: a.petzold@ion.ucl.ac.uk

†Neurology Service, Centre Hospitalier Universitaire Vaudois \& University of Lausanne, Switzerland

$\ddagger$ Pain Relief Unit, Department of Anaesthesiology, University of Wuerzburg, Wuerzburg, Germany.

$\S$ Neurology Service, Centre Hospitalier Universitaire Vaudois \& University of Lausanne, Switzerland 


\begin{abstract}
Body fluid biomarkers of CNS damage may help improving the prognostic and diagnostic accuracy in ischaemic stroke.

Fifty-three patients were included. Stroke severity and outcome was rated using the NIHSS and modified Ranking Scale (mRS). Ferritin, S100B and $\mathrm{NfH}$ were measured in CSF and serum. Infarct volume was calculated from T2W images.

CSF S100B (median $1.00 \mathrm{ng} / \mathrm{mL}$ ) and CSF ferritin $(10.0 \mathrm{ng} / \mathrm{mL}$ ) levels were elevated in stroke compared to the controls $(0.62 \mathrm{ng} / \mathrm{mL}$, $\mathrm{p}<0.0001 ; 2.34 \mathrm{ng} / \mathrm{mL}, \mathrm{p}<0.0001)$. Serum S100B $(0.09 \mathrm{ng} / \mathrm{mL})$ was higher in stroke compared to the controls $(0.01 \mathrm{ng} / \mathrm{mL})$. CSF S100B levels were higher in patients with a cardio-embolic stroke $(2.88 \mathrm{ng} / \mathrm{mL})$ than in those with small vessel disease $(0.89 \mathrm{ng} / \mathrm{mL}, p<0.05)$. CSF S100B levels correlated with the NIHSS on admission $(R=0.56, p<0.01)$ and the stroke volume $(\mathrm{R}=0.44, \mathrm{p}=0.01)$. CSF S100B and NfH-SMI35 levels correlated with outcome on the mRS.

CSF S100B levels were related to stroke severity and infarct volume and highest in in cardio-embolic stroke.
\end{abstract}




\section{Introduction}

Ischaemic stroke is one of the major causes of death and disability in the Western population over 60 years of age. ${ }^{1}$ Transient or permanent occlusion of a blood vessel leads to ischaemic damage to the glial and nerve cells in the affected vascular territory. There is evidence that glial cells may be more susceptible to ischaemia than neurons. ${ }^{2-4}$ Following cellular disintegration, cell-type specific proteins are released into the extracellular fluid (ECF). These proteins pass from the ECF into the cerebrospinal fluid (CSF) and blood, from where they can be quantified. In addition to the immediate release of these proteins from dying cells, some proteins such as the cytokine S100B will also be actively secreted by glial cells in response to the brain's homeostatic imbalance. Similarly ferritin, an ironbinding protein, will be released by microglia in the presence of iron. Several groups have recently called into question the validity of blood S100B levels as a specific biomarker for brain damage ${ }^{5-8}$ whereas others have confirmed it. 9,10 The main argument of the former group is that $\mathrm{S100B}$ is not present exclusively in astrocytes ${ }^{11}$ but can also be measured in the blood of patients who did not suffer from brain injury, such as patients with critical illness ${ }^{8}$ and those with systemic trauma. ${ }^{12,13}$ In contrast, CSF S100B levels reflect more accurately the pathological changes in the cen- 
tral nervous system (CNS). ${ }^{7}$ In fact, serum and CSF S100B levels did not correlate in one study. ${ }^{14}$ Given the number of studies on levels of serum S100B in stroke patients, ${ }^{15-24}$ there is surprisingly little literature on CSF S100B levels in patients with a well-defined ischaemic stroke. A Medline search on CSF and S100 revealed 154 hits (25-JUN-2007), yet none was focused on stroke. Four studies referred to patients with ischaemic brain injury without providing more clinical details. ${ }^{11,25-27}$ One patient described by Anderson et al. developed a stroke following surgery for an aneurysm of the thoracoabdominal aorta. ${ }^{28}$ To the best of our knowledge, there are no published studies on combined CSF and serum S100B levels in a well defined cohort of patients with an ischaemic cerebrovascular event.

This study was therefore designed to bridge the gap between data on S100B in the CSF and the serum. We hypothesised that the previously reported relationship between serum biomarker levels ${ }^{20,22}$ and infarct volume, stroke severity and outcome would also exist for CSF levels of the same biomarkers.

\section{Methods}

Patients Serum and CSF from 33 patients admitted to the Centre Hospitalier Universitaire Vaudois (CHUV) Lausanne with suspicion of ischaemic stroke was collected prospectively and kept in a hospital CSF bank. All 
clinical and radiological data was collected prospectively with the clinician being blinded to the data on the CSF and serum biomarkers. In all cases the indication for performing a lumbar puncture (LP) was to exclude vasculitis. All patients were assessed clinically using the National Institutes of Health stroke scale (NIHSS) and the modified Rankin scale (mRS). Clinical outcome was assessed with the mRS at discharge. An mRS score of 3 to 6 was considered a poor outcome. The study has been approved by the appropriate local ethics committee and was therefore performed in accordance with the ethical standards laid down in the 1964 Declaration of Helsinki.

The control group consisted of 20 non-neurological patients ( 8 female, 12 male) receiving spinal anesthesia for minor surgery. The median age of the control group was 33.5 years (IQR 24-54 years).

Sample collection and analysis The CSF samples were collected and time-matched. The samples were coded and anonymised. The CSF was centrifuged $(2500 \mathrm{rpm})$ and the supernatant stored at $-80^{\circ} \mathrm{C}$ until analysis.

The concentrations of $\mathrm{S} 100 \mathrm{~B}$, ferritin and $\mathrm{NfH}^{S M I 35}$ were analyzed using previously-described ELISA techniques. ${ }^{14,29-31}$ The analyst was blinded to all other clinical or radiological information. All data were exchanged between the centers prior to breaking the code. 
Reference values For rigorism this study used previously published reference limits in addition to the control group. The control group of nonneurological patients allowed for quantification of all three biomarkers from the same CSF sample, which was assumed to be completely normal.

However, the use CSF from neurologically normal patients does potentially introduce a bias with artificially low CSF biomarker levels, with a statistical significant but clinically irrelevant result. For this reason we made additional use of the previously published normal ranges for the CSF biomarkers, which were derived from our own large reference populations ( $n=388$ to $\left.416^{14,29,31}\right)$ using exactly the same in-house developed assays. If CSF biomarkers were above the published upper limit of normal then they were regarded as pathological (high).

MRI analysis MRI for final infarct volume calculation was usually performed within 2 weeks of symptom onset using a Siemens Magnetom Vision 1.5 Tesla MRI-scanner with echo-planar imaging. T2-images were obtained using turbo spin echo, with a matrix of $192 \times 256$, slice thickness of $5 \mathrm{~mm}$, echo/repetition time of $3800 / 90$, and a gap of $1.5 \mathrm{~mm}$. The infarcted cerebral areas on the T2W images were manually traced on each slice. These values were then added and multiplied by the slice-thickness in order to obtain volumetric values which were expressed in cubic cen- 
timeters $(\mathrm{cm} 3)$. In four patients, final stroke volumes were calculated using plain CT between day 2 and 14 after stroke onset. The radiological assessment was done blinded to any of the clinical or biomarker data.

Statistical analysis Statistical analysis was performed using SAS software. Independent variables were compared using the non-parametric two-sample exact Wilcoxon rank-sum test for two variables and a two-way unbalanced ANOVA (general linear model) for more than two variables, followed by multivariate analysis when appropriate. The linear relationship between continuous variables was evaluated using the Spearman correlation coefficient. The ordinal NIHSS was treated as a linear variable in the regression models. The level of significance for the multiple correlations was corrected using the Bonferroni method. The $\chi^{2}$-test and Fisher's exact test were used for comparing proportions of patients. Two-tailed tests were used throughout and a $p$-value of $<0.05$ was accepted as significant.

\section{Results}

The patient characteristics are presented in Table 1. In addition to ischaemic stroke due to cardio-embolic, large- or small vessel disease, there was one patient each with $p-A N C A$ vasculitis $(n=1)$, herpes zoster vasculitis $(n=1)$, anti-phospholipid antibodies $(n=1)$ and malignancy $(n=1)$. 
There was no correlation of the patients age with either the NIHSS on admission or at discharge, or with the mRS.

CSF biomarker levels The CSF S100B $(p<0.0001)$ and ferritin $(p<0.0001)$ levels were significantly higher in patients suffering from a stroke when compared to the non-neurological control group (Table 2). No such difference was found for CSF $\mathrm{NfH}^{S M I 35}$ levels.

These results were confirmed using our upper reference values (Table 3). Eighty-two percent of the stroke patients had elevated CSF S100B levels. Thirty-nine percent of the stroke patients had elevated CSF ferritin levels. One patient suffered from a haemorrhagic transformation two days after his ischaemic stroke and the LP was performed about 24 hours after the event. This patient had CSF ferritin levels over 10-fold higher (150 $\mathrm{ng} / \mathrm{mL}$ ) than the upper reference limit $(12 \mathrm{ng} / \mathrm{mL})$. Only $7 \%$ of the stroke patients had elevated CSF NfH ${ }^{\text {SMIS5 }}$ levels.

There were no correlations between any of the CSF biomarkers with age or the time delay from onset of stroke to lumbar puncture (median 3 days, range 1 to 15 days). The median CSF total protein, CSF:serum albumin quotient $\left(\mathrm{Q}_{a l b}\right.$, white and red cell count were in the normal range (Table 1). An elevated total protein was found in 12, an elevated $Q_{a l b}$ in 14, an elevated white cell count in 3 and an elevated red cell count in 9 
patients. There were no correlations between any of the CSF biomarker with either the CSF total protein, $\mathrm{Q}_{a l b}$, CSF white or red cell count.

Serum biomarker levels The median serum S100B levels were higher in patients with stroke compared to non-neurological controls $(p<0.0001)$. In contrast serum $\mathrm{NfH}^{S M I 35}$ levels were higher in the control group $(\mathrm{p}<0.05)$.

As for the CSF, there were no correlations between any of the serum biomarkers with age or the time delay from onset of stroke to venopuncture.

Relationship between CSF and serum biomarkers In stroke patients, there was a weak correlation between the CSF $\mathrm{NfH}^{S M I 35}$ and serum levels $(R=0.40, p=0.03$ ), but not between the CSF and the serum S100B levels ( $R=0.14, p=0.42$ ). In the control group there was no correlation between CSF and serum levels for either S100B $(R=0.02, p=0.9)$ or $\mathrm{NfH}^{S M I 35}(\mathrm{R}=0.05$, $\mathrm{p}=0.8)$.

Aetiology Because of small numbers for some causes (see Table 1), this particular study included only patients with cardio-embolic, and largeand small-vessel disease (Table 1).

There was a significant difference in CSF S100B levels between the three main causes $\left(F_{2,20}=4.5, p=0.025\right)$. The post-hoc analysis showed 
this to be caused by significantly higher CSF S100B levels in patients with cardio-embolic stroke (median $2.88 \mathrm{ng} / \mathrm{mL}$ ) compared to those with smallvessel disease $(0.89 \mathrm{ng} / \mathrm{mL}, \mathrm{p}=0.014$, Figure 1$)$ and large-vessel disease (0.44 ng/mL, $\mathrm{p}=0.031)$. A multivariate analysis was also performed because cardio-embolic strokes are known to have a larger infarct volume than those from small-vessel disease. Importantly, the significance remained after correcting for infarct volume $(F=10.2, p=0.017)$. In contrast to the CSF S100B levels, the stroke volume alone did not distinguish between the main aetiologies $\left(\mathrm{F}_{2,20}=3.3, \mathrm{p}=0.06\right)$.

Similar to the $\mathrm{CSF}$, serum $\mathrm{NfH}^{S M I 35}\left(\mathrm{~F}_{2,20}=5.04, \mathrm{p}=0.017\right)$ but not serum S100B distinguished between the main aetiologies. The post-hoc analysis demonstrated that serum $\mathrm{NfH}^{S M I 35}$ levels were significantly higher in patients with stroke due to large-vessel pathology (median $0.15 \mathrm{ng} / \mathrm{mL}$ ) when compared to those with small-vessel pathology (median $0.00 \mathrm{ng} / \mathrm{mL}$, $p=0.004$ ). However, in contrast to the finding for CSF S100B levels, significance was lost for the serum S100B levels after correcting for infarct volume $(F=0.07, p=0.93)$.

Vascular territory The affected vascular territories are shown in Table 1. There was no relationship between the affected vascular territory and the CSF and serum S100B, ferritin or $\mathrm{NfH}^{S M I 35}$ levels (data not shown). 
Stroke volume The median volume of all strokes was $4 \mathrm{~cm} 3$ (see Table 1). The ischaemia volume correlated weakly with the CSF S100B levels $(R=0.44, p=0.01)$ and the NIHSS on admission $(R=0.56, p<0.01)$. The data are shown in Figure 2 on a logarithmic scale because of a cluster of small volume strokes $(<5 \mathrm{~cm} 3)$. The smallest stroke volume related to high CSF S100B levels (>0.55 ng/mL) was $0.5 \mathrm{~cm} 3$. The other biomarkers were not related to stroke volume.

Stroke severity on admission The median NIHSS score on admission was 4 (see Table 1). There was a significant correlation between CSF S100B levels and the NIHSS on admission $(R=0.56, p<0.001$, Figure 3$)$. No such correlation with the NIHSS was found for CSF $\mathrm{NfH}^{S M I 35}$ or ferritin levels or the serum levels of S100B and $\mathrm{NfH}^{\text {SMI35 }}$.

Outcome As expected, there was a strong correlation between the NIHSS on admission with the NIHSS at discharge $(R=0.77, p<0.0001)$ and the $m R S$ at discharge $(R=0.75, p<0.0001)$.

With regard to the biomarkers, a weak correlation with the mRS on discharge was found with the CSF $\mathrm{NfH}^{S M I 35}(\mathrm{R}=0.37, \mathrm{p}=0.049)$ and $\mathrm{CSF}$ S100B levels $(R=0.36, p=0.039)$ as well as with the stroke volume $(R=0.39$, $\mathrm{p}=0.026$ ). The degree of improvement in the NIHSS did correlate inversely 
with the CSF S100B levels $(R=-0.5, p=0.0035)$.

Because the samples were collected at different time points, a partial correlation with the time delay as a co-variate was performed. Significance remained for the correlation between the mRS on discharge and CSF S100B levels $(R=0.49, p=0.01)$ and the stroke volume $(R=0.40, p=0.039)$, but was lost for $\mathrm{CSF} \mathrm{NfH}{ }^{S M I 35}(\mathrm{R}=0.35, \mathrm{p}=0.07)$. As before, patients with higher CSF S100B levels showed less improvement on the NIHSS than those with lower CSF S100B levels $(R=-0.45, p=0.018)$.

\section{Discussion}

The main findings of this study were increased levels of S100B and ferritin in the CSF of a substantial number of patients suffering from an ischaemic stroke. Increased CSF S100B levels were found in $82 \%$ of these patients and were significantly higher than in the control population. The highest levels were present in patients who suffered from an ischaemic cardioembolic stroke (Figure 1 and Table 3). CSF S100B levels did correlated with the clinical severity of stroke on admission (NIHSS, R=0.56, Figure 3). Additionally, CSF S100B levels but not serum S100B levels were related to outcome expressed either as the level of improvement on the NIHSS or as the mRS on discharge. Although this suggests that CSF S100B levels may be more sensitive than serum S100B levels, it needs to be 
borne in mind that most patients in the present study only suffered from a minor ischaemic stroke, and a larger amount of CNS tissue destruction may have brought to light a relationship between the serum S100B levels and outcome as demonstrated by others who investigated patients with a more severe ischaemic stroke. ${ }^{19-22,32,33}$

An increase in CSF ferritin levels was observed in 39\% of patients with an ischaemic stroke (Table 3). The highest level was found in the CSF of one patient two days following a haemorrhagic transformation (CSF ferritin $150 \mathrm{ng} / \mathrm{mL}$, CSF total protein $1.5 \mathrm{~g} / \mathrm{L}$, CSF red cells 2768 , CSF white cells 23). There is as yet no literature available on longitudinal ferritin levels in the CSF of patients who have suffered haemorrhagic strokes. The biological function of ferritin is to bind and transport iron. Ferritin is actively produced by microglia in response to any free iron within the central nervous system. Following an intracranial or subarachnoid bleed erythrocytes haemolyse and the released haemoglobin is metabolised. During this process iron is liberated, ferritin binds this toxic element and delivers it back to the intra cellular department. ${ }^{34} \mathrm{CSF}$ ferritin levels appear to be a reliable indicator of a haemorrhagic transformation, but with the increasing availability of brain imaging facilities, the clinical use may only be relevant to some selected cases.

There was no evidence for an increase in $\mathrm{CSF} \mathrm{NfH}^{S M I 35}$ levels in pa- 
tients with ischaemic strokes. There are two potential reasons for this negative finding. Firstly, the location of the stroke may have been too rostral to have been reflected in lumbar CSF. However, after reviewing all brain scans, CSF $\mathrm{NfH}^{S M I 35}$ levels were similar in those patients with predominantly cortical to those with predominantly subcortical infarcts (data not shown). CSF NfH ${ }^{S M I S 5}$ levels showed a weak correlation with the modified Rankin scale at discharge. This may be a spurious statistical finding because CSF $\mathrm{NfH}^{S M I 35}$ levels remained within the normal reference range (Tables 2 and 3). Furthermore, the significance of the finding was lost after correcting for the time delay between the stroke and the LP. Likewise the slightly higher serum $\mathrm{NfH}^{S M I 35}$ levels in the control group compared to the ischaemic stroke patients may be due to an intravenous fluid related dilution effect in the stroke patients.

An intriguing new finding of this study was that the higher CSF S100B levels in patients with an ischaemic cardio-embolic stroke compared to those with large- or small-vessel disease were independent from stroke volume. Our results therefore suggest that the damage to the CNS may either be more widespread, more diffuse than shown by routine T2W brain imaging or the consequence of ongoing micro-embolic infarctions. Interestingly, a recent study on S100B in patients with dilated cardiomyopathy found the mean serum levels to be about 4-times higher in patients $(0.017$ 
$\mathrm{ng} / \mathrm{mL})$ compared to controls $(0.051 \mathrm{ng} / \mathrm{mL}, \mathrm{p}<0.01) .{ }^{35}$ Inspection of the raw data in Figure 2 in reference ${ }^{35}$ shows that this was due to a cluster of seven of the 21 cardiomyopathy patients having serum S100B levels above $0.1 \mathrm{ng} / \mathrm{mL}$. Based on a weak correlation of serum S100B with the $\mathrm{N}$ terminal portion of the brain natriuretic peptide pro-hormone (NT-proBNP) it is suggested that serum S100B may be of cardiac origin, but the authors are careful not to exclude the possibility of the brain parenchyma being an alternative source. Our data on CSF instead of serum S100B levels suggests that at least in our study S100B is released from the brain parenchyma. Additionally the correlation between S100B and NT-proBNP is not entirely convincing. Inspection of the raw data in Figure 3 in reference $^{35}$ shows that these seven cardiomyopathy patients with high serum S00B levels were spread across the whole range of NT-proBNP with some of the highest S100B values being actually in the lower NT-proBNP range, with no such correlation in the control group. Because of the epidemiological demonstrated association between cardiomyopathy with arterial fibrillation (AF) and cardio-embolic stroke the present finding of higher CSF S100B levels in patients with cardio-embolic stroke need to be investigated further. A limitation is that neither the present study nor the study by Mazzini et al. ${ }^{35}$ did include any data on AF. Another limitation is the small number in the present study with only 9 of the 33 stroke patients suf- 
fering from a cardio-embolic stroke. Based on our data 28 patients with a stroke due to cardio-embolic aetiology or small-vessel disease need to be recruited in any future study to reach a power of $80 \%$ (alpha 0.05 ) to test this hypothesis. Diffusion weighted imaging which is more sensitive than T2W imaging may also be of help in future studies to address this question. Cardio-embolic stroke accounts for about $19 \%$ of ischaemic strokes. ${ }^{36}$ As secondary stroke prevention continues to evolve ${ }^{37}$, it may be interesting to measure blood S100B levels prospectively, alongside blood glucose, cholesterol, etc. in the longitudinal follow-up of these patients. The correlation of CSF S100B levels with infarct volume is consistent to that described for serum S100B levels. ${ }^{20,22}$ Likewise the correlation of CSF S100B levels with stroke severity on admission is in line with previous results on serum S100B levels. ${ }^{20,22}$

Consistent with our previous data on patients with traumatic brain injury and subarachnoid haemorrhage, ${ }^{14}$ yet again there was no correlation between the CSF and serum levels of S100B. Likewise there was no correlation between CSF and serum $\mathrm{NfH}^{S M I 35}$ levels. The most likely reason for a lack of a linear correlation is that the biological half-life of S100B and $\mathrm{NfH}^{\text {SMI35 }}$ differs between the CSF and the blood. The physiological "washout" pattern of these two protein biomarkers from the CSF compartment in the serum is not known. From the perfusion study in melanoma patients 
by Ghanem et al. ${ }^{38}$ we know that the half-life of $\mathrm{S} 100 \mathrm{~B}$ in the serum is about 30 minutes. This is considerably shorter then the half life of $\mathrm{S} 100 \mathrm{~B}$ in the CSF of 2 to 3 days observed in subarachnoid haemorrhage or 1 to 2 days in intracranial haemorrhage (Figure 1 in reference ${ }^{14}$ ). The half-life of S100B in the CSF in patients with stroke is not known. Additionally the situation may be complicated by the development of secondary brain injury or persistent astrocytic activation which may cause a secondary increase of CSF S100B levels. ${ }^{39}$ New insight in dynamics of these processes can be gained using microdialysis which allows for frequent sampling of extracellular fluid (ECF) from the brain parenchyma. Our own experience is that ECF S100B raises rapidly in response to raise of intracranial pressure or cerebral vasospasm. ${ }^{40}$ The data also shows that the half-life of S100B in the ECF is less then one hour. The present study does not explain how these protein biomarkers are "washed-out" it makes the important point that there was no linear correlation which in all likelihood also applies to other protein biomarkers currently investigated in the blood of stroke patients.

Further limitations of our study are the relatively young median age of our patients, and their low NIHSS on admission. Although we did not find a correlation between the biomarker levels and the time delay to CSF or serum collection, we cannot exclude that this may have influenced the 
described relationships. Future longitudinal studies may enable this caveat to be addressed.

Another important limitation of the present study is that the median delay from onset of the ischaemic stroke to sampling time was relatively long, with a median of 3 days and a range of up to 15 days. This is considerably longer than that achieved in studies on serum S100B levels with 3 hours, ${ }^{32}$ 5 hours, ${ }^{19} 6$ hours, ${ }^{20} 8$ hours ${ }^{21}$ or 24 hours $^{22}$ in some of the more recent studies. Given the previously-described wash-out pattern of S100B from the CSF into the blood ${ }^{14}$, one would expect to find higher CSF S100B levels during the first hours following the ischaemic stroke. Therefore this study does not rule out very early rises in S100B as a good indicator of the amount of primary brain injury and thus its use as an important prognostic indicator. Additionally, this study is weakened by the collection of samples at different time points following the stroke. Given the well established longitudinal profile for serum S100B levels ${ }^{19-22,32,33}$, one would be hesitant to generalise from the present data. However, serial CSF sampling in ischaemic stroke is not feasible. We do not believe that CSF sampling will have a major place in the diagnostic workup of most ischaemic stroke patients. However, the present result showing a lack of correlation between CSF and serum biomarker levels in stroke is important for future biomarker studies from the blood. It suggests that future studies on serum 
biomarkers need to be conducted longitudinally, ideally in short time intervals, in order to account for the unknown release and wash out pattern of biomarkers from the brain parenchyma following an ischaemic stroke.

\section{Acknowledgments and Funding}

P. Michel has received speaker and consulting fees from Biosite Inc, San Diego, California. The other authors have nothing to declare. The authors are thankful to Hugues Henry, PhD, for his collaboration in the organisation of the CSF bank and Drs Robert Schedel, Guenter Sprotte, Heiko Vogel, Manuela Rott, Christine Melzer for help with sample collection.

This study was presented at the 17th meeting of the European Neurological Society (ENS) in Rhodes, June 16-20 2007. 


\section{References}

[1] PA Wolf. Stroke: Pathophysiology, Diagnosis, and Management, chapter Epidemiology of stroke, pages 13-34. Churchill Livingstone, 4th edition, 2004.

[2] T. Sugawara, A. Lewen, N. Noshita, Y. Gasche, and P.H. Chan. Effects of global ischemia duration on neuronal, astroglial, oligodendroglial, and microglial reactions in the vulnerable hippocampal CA1 subregion in rats. J Neurotrauma, 19:85-98, 2002.

[3] L.L. Dugan and J.S. Kim-Han. Astrocyte mitochondria in in vitro models of ischemia. J Bioenerg Biomembr, 36:317-321, 2004.

[4] RG Giffard, MC Papadopoulos, JA van Hooft, L Xu, R Giuffrida, and Monyer $\mathrm{H}$. The electrogenic sodium bicarbonate cotransporter: developmental expression in rat brain and possible role in acid vulnerability. J Neursci, 20:1001-1008, 2000.

[5] J. Steiner, H.G. Bernstein, H. Bielau, A. Berndt, R. Brisch, C. Mawrin, G. Keilhoff, and B. Bogerts. Evidence for a wide extra-astrocytic distribution of S100B in human brain. BMC Neurosci, 8:2, 2007.

[6] A.B. da Rocha, R.F. Schneider, G.R. de Freitas, C. Andre, I. Grivicich, C. Zanoni, A. Fossa, J.T. Gehrke, G. Pereira Jotz, M. Kaufmann, D. Simon, and A. Regner. Role of serum S100B as a predictive marker of fatal outcome following isolated severe head injury or multitrauma in males. Clin Chem Lab Med, 44:1234-1242, 2006.

[7] A. Kleindienst and M. Ross Bullock. A critical analysis of the role of the neurotrophic protein S100B in acute brain injury. J Neurotrauma, 23:1185-1300, 2006.

[8] C. Routsi, E. Stamataki, S. Nanas, C. Psachoulia, A. Stathopoulos, A. Koroneos, M. Zervou, G. Jullien, and C. Roussos. Increased levels of serum S100B protein in critically ill patients without brain injury. Shock, 26:20-4, 2006.

[9] J.R. Lynch, R. Blessing, W.D. White, H.P. Grocott, M.F. Newman, and D.T. Laskowitz. Novel diagnostic test for acute stroke. Stroke, 35:5763, 2004.

[10] S. Korfias, G. Stranjalis, A. Papadimitriou, C. Psachoulia, G. Daskalakis, A. Antsaklis, and D. E. Sakas. Serum S-100B protein 
as a biochemical marker of brain injury: a review of current concepts. Curr Med Chem, 13(30):3719-31, 2006.

[11] CJ Sindic, MP Chalon, CL Cambiaso, EC Laterre, and PL Masson. Assessment of damage to the central nervous system by determination of S-100 protein in the cerebrospinal fluid. J Neurol Neurosurg Psychiatry, 45:1130-1105, 1982.

[12] J. Unden, J. Bellner, M. Eneroth, C. Alling, T. Ingebrigtsen, and B. Romner. Raised serum S100B levels after acute bone fractures without cerebral injury. J Trauma, 58:59-61, 2005.

[13] O. Savola, J. Pyhtinen, T.K. Leino, S. Siitonen, O. Niemela, and M. Hillbom. Effects of head and extracranial injuries on serum protein S100B levels in trauma patients. J Trauma, 56:1229-1234, 2004.

[14] A Petzold, G Keir, D Lim, M Smith, and EJ Thompson. CSF and serum S100B: release and wash-out pattern. Brain Res Bull, 61:281285, 2003.

[15] HD Abraha, RJ Butterworth, PM Bath, WS Wassif, J Garthwaite, and RA Sherwood. Serum S-100 protein, relationship to clinical outcome in acute stroke. Ann Clin Biochem, 34:546-550, 1997.

[16] T. Buttner, S. Weyers, T. Postert, R. Sprengelmeyer, and W. Kuhn. S-100 protein: serum marker of focal brain damage after ischemic territorial MCA infarction. Stroke, 28:1961-1905, 1997.

[17] J.W. Elting, A.E. de Jager, A.W. Teelken, M.J. Schaaf, N.M. Maurits, J. van der Naalt, C.T. Sibinga, G.A. Sulter, and J. De Keyser. Comparison of serum S-100 protein levels following stroke and traumatic brain injury. J Neurol Sci, 181:104-10, 2000.

[18] U. Missler, M. Wiesmann, C. Friedrich, and M. Kaps. S-100 protein and neuron-specific enolase concentrations in blood as indicators of infarction volume and prognosis in acute ischemic stroke. Stroke, 28:1956-1960, 1997.

[19] C Foerch, R Du Mesnil De Rochemont, O Singer, T NeumannHaefelin, M Buchkremer, FE Zanella, H Steinmetz, and M Sitzer. $\mathrm{S} 100 \mathrm{~B}$ as a surrogate marker for successful clot lysis in hyperacute middle cerebral artery occlusion. J Neurol Neurosurg Psychiatry, 74:322-305, 2003. 
[20] M.T. Wunderlich, C.W. Wallesch, and M. Goertler. Release of neurobiochemical markers of brain damage is related to the neurovascular status on admission and the site of arterial occlusion in acute ischemic stroke. J Neurol Sci, 227:49-53, 2004.

[21] C. Foerch, B. Otto, O.C. Singer, T. Neumann-Haefelin, B. Yan, J. Berkefeld, H. Steinmetz, and M. Sitzer. Serum S100B predicts a malignant course of infarction in patients with acute middle cerebral artery occlusion. Stroke, 35:2160-2104, 2004.

[22] C. Foerch, O.C. Singer, T. Neumann-Haefelin, R. du Mesnil de Rochemont, H. Steinmetz, and M. Sitzer. Evaluation of serum S100B as a surrogate marker for long-term outcome and infarct volume in acute middle cerebral artery infarction. Arch Neurol, 62:1130-1104, 2005.

[23] L Persson, HG Hardemark, J Gustafsson, G Rundstrom, I MendelHartvig, T Esscher, and S Pahlman. S-100 protein and neuronspecific enolase in cerebrospinal fluid and serum: markers of cell damage in human central nervous system. Stroke, 18:911-908, 1987.

[24] A Aurell, LE Rosengren, B Karlsson, et al. Determination of S-100 and glial fibrillary acid protein concentration in cerebrospinal fluid after brain infarction. Stroke, 22:1254-1258, 1991.

[25] KJ Lamers, BG van Engelen, FJ Gabreels, OR Hommes, GF Borm, and RA Wevers. Cerebrospinal neuron-specific enolase, S-100 and myelin basic protein in neurological disorders. Acta Neurol Scand, 92:247-251, 1995.

[26] K Mokuno, K Kato, K Kawai, Y Matsuoka, T Yanagi, and I Sobue. Neuron-specific enolase and S-100 protein levels in cerebrospinal fluid of patients with various neurological diseases. J Neurol Sci, 60:443-451, 1983.

[27] F Michetti, A Massaro, G Russo, and G Rigon. The S-100 antigen in cerebrospinal fluid as a possible index of cell injury in the nervous system. Neurol Sci, 44:259-263, 1980.

[28] R.E. Anderson, A. Winnerkvist, L.O. Hansson, O. Nilsson, L. Rosengren, G. Settergren, and J. Vaage. Biochemical markers of cerebrospinal ischemia after repair of aneurysms of the descending and 
thoracoabdominal aorta. J Cardiothorac Vasc Anesth, 17:598-603, 2003.

[29] A Petzold, G Keir, AJE Green, G Giovannoni, and EJ Thompson. A specific ELISA for measuring neurofilament heavy chain phosphoforms. J Immunol Methods, 278:179-190, 2003.

[30] AJE Green, G Keir, and EJ Thompson. A specific and sensitive ELISA for measuring S-100b in cerebrospinal fluid. J Immunol Meth, 205:3541, 1997.

[31] G Keir, N Tasdemir, and EJ Thompson. Cerebrospinal-fluid ferritin in brain necrosis - evidence for local synthesis. Clin Chim Acta, 216:153-166, 1993.

[32] M.T. Wunderlich, C.W. Wallesch, and M. Goertler. Release of glial fibrillary acidic protein is related to the neurovascular status in acute ischemic stroke. Eur J Neurol, 13:1118-1123, 2006.

[33] M. Stroick, M. Fatar, A. Ragoschke-Schumm, K. Fassbender, T. Bertsch, and M.G. Hennerici. Protein S-100B-a prognostic marker for cerebral damage. Curr Med Chem, 13:3053-3060, 2006.

[34] P.J. Sargent, S. Farnaud, and R.W. Evans. Structure/function overview of proteins involved in iron storage and transport. Curr Med Chem, 12:2683-2693, 2005.

[35] G. S. Mazzini, D. V. Schaf, E. R. Vinade, E. Horowitz, R. S. Bruch, L. M. Brunm, C. A. Goncalves, F. Bacal, D. O. Souza, L. V. Portela, and S. Bordignon. Increased S100B serum levels in dilated cardiomyopathy patients. J Card Fail, 13:850-4, 2007.

[36] D.J. McCabe and R.D. Rakhit. Antithrombotic and interventional treatment options in cardioembolic transient ischaemic attack and ischaemic stroke. J Neurol Neurosurg Psychiatry, 78:14-24, 2007.

[37] M. Girot, M.A. Mackowiak-Cordoliani, D. Deplanque, H. Henon, C. Lucas, and D. Leys. Secondary prevention after ischemic stroke. Evolution over time in practice. J Neurol, 252:14-20, 2005.

[38] G. Ghanem, B. Loir, R. Morandini, F. Sales, D. Lienard, A. Eggermont, and F. Lejeune. On the release and half-life of S100B protein in the peripheral blood of melanoma patients. Int J Cancer, 94:586-90, 2001. 
[39] A Petzold. CSF biomarkers for improved prognostic accuracy in acute CNS disease. Neurological Research, 29:691-708, 2007.

[40] J Sen, A Belli, A Petzold, et al. Extracellular S100B in the injured brain: a highly sensitive surrogate marker for acute brain injury. Acta Scand Neuropathol, 147:897-900, 2005. 
Table 1: Characteristics of the patients. For aetiology of the ischaemic stroke in patients labeled as "other" see main text.

\begin{tabular}{lll}
\hline Characteristic & Median / \% & Range / number \\
\hline Age (years) & 53 & $33-79$ \\
Gender (F:M) & $48 \%: 52 \%$ & $16: 17$ \\
Time from CVA to LP (days) & 3 & $1-15$ \\
NIHSS score & & \\
on admission & 4 & $0-13$ \\
at discharge & 1 & $0-11$ \\
modified Rankin score & & \\
at discharge & 2 & $0-5$ \\
Aetiology & & \\
cardiac & $26 \%$ & $9 / 33$ \\
large-vessel disease & $11 \%$ & $4 / 33$ \\
small-vessel disease & $26 \%$ & $9 / 33$ \\
other & $11 \%$ & $4 / 33$ \\
unknown & $24 \%$ & $8 / 33$ \\
Artery & & \\
Basilar & $15 \%$ & $5 / 33$ \\
Middle cerebral & $52 \%$ & $17 / 33$ \\
Posterior cerebral & $3 \%$ & $1 / 33$ \\
Vertebral & $9 \%$ & $3 / 33$ \\
Multiple & $9 \%$ & $3 / 33$ \\
Lacunar & $12 \%$ & $4 / 33$ \\
Infarct volume (cm3) & 4 & $0-78$ \\
CSF & & \\
Total protein (g/L) & & $0.27-1.53$ \\
Q ${ }_{\text {alb }}$ & 0.43 & $3.9-28.6$ \\
White cell count mm ${ }^{3}$ & 6.6 & $0-23$ \\
Red cell count $\mathrm{mm}^{3}$ & 10 & \\
\hline
\end{tabular}

\footnotetext{
${ }^{1}$ Reference range $0.15-0.45 \mathrm{mg} / \mathrm{L}$
} 
Table 2: CSF and serum levels of S100B [ng/mL] and $\mathrm{NfH}^{S M I 35}[\mathrm{ng} / \mathrm{ml}]$ and $\mathrm{CSF}$ ferritin $[\mathrm{ng} / \mathrm{mL}]$ levels in patients with an ischaemic stroke and the control cohort. N.S. = not significant. The median (IQR) is shown.

\begin{tabular}{llll}
\hline Biomarker $[\mathrm{ng} / \mathrm{mL}]$ & Controls & Stroke & $\mathrm{p}-$ value \\
\hline CSF S100B median (range) & $0.62(0.51-0.70)$ & $1.00(0.62-1.52)$ & $<0.01$ \\
Serum S100B median (range) & $0.01(0.00-0.03)$ & $0.09(0.07-0.11)$ & $<0.0001$ \\
& & & \\
CSF NfH ${ }^{S M I 35}$ median (range) & $0.20(0.09-0.24)$ & $0.10(0.07-0.16)$ & $\mathrm{N} . S$. \\
Serum NfH & & \\
& & & \\
CSF ferritin median (range) & $2.34(1.59-3.45)$ & $10.0(6.0-15.0)$ & $\mathrm{p}<0.0001$ \\
\hline
\end{tabular}


Table 3: CSF levels of S100B, NfH ${ }^{S M I 35}$ and ferritin in patients with an ischaemic stroke and the respective, previously published reference populations. ${ }^{14,29,31}$ Levels above the published upper reference limit are indicated as 'high' and levels within the reference range as 'normal'. N.S. = not significant (two-sided Fisher's exact test).

\begin{tabular}{llll}
\hline & Reference population & Stroke patients & Significance \\
\hline S100B high & $4(1 \%)$ & $27(82 \%)$ & \\
S100B normal & $405(99 \%)$ & $6(18 \%)$ & $\mathrm{p}<0.0001$ \\
& & $2(7 \%)$ & \\
$\mathrm{NfH}^{S M I 35}$ high $^{3}$ & $20(5 \%)$ & $27(93 \%)$ & N.S. \\
$\mathrm{NfH}^{S M I 35}$ normal & $396(95 \%)$ & & \\
& & $11(39 \%)$ & \\
Ferritin high & $20(5 \%)$ & $17(61 \%)$ & $\mathrm{p}<0.0001$ \\
Ferritin normal & $386(95 \%)$ & & \\
\hline
\end{tabular}

\footnotetext{
${ }^{2}$ The upper reference limit of $0.55 \mathrm{ng} / \mathrm{mL}$ was 99 th percentile of the cumulative frequency of the results from 409 patients ( 233 female, 176 male) with a mean age of 46 years (range 20 to 99 years). ${ }^{14}$

${ }^{3}$ The upper reference limit of $0.73 \mathrm{ng} / \mathrm{mL}$ was 95 th percentile of the cumulative frequency of the results from 416 patients ( 242 female, 166 male, 8 without gender information) with a mean age of 42 years (interquartile range $31.2-5.8$ years). ${ }^{29}$

${ }^{4}$ The upper reference limit of $12 \mathrm{ng} / \mathrm{mL}$ was determined as the 95 th percentile of the cumulative frequency of the results from 388 patients (no further demographic data available). ${ }^{31}$
} 


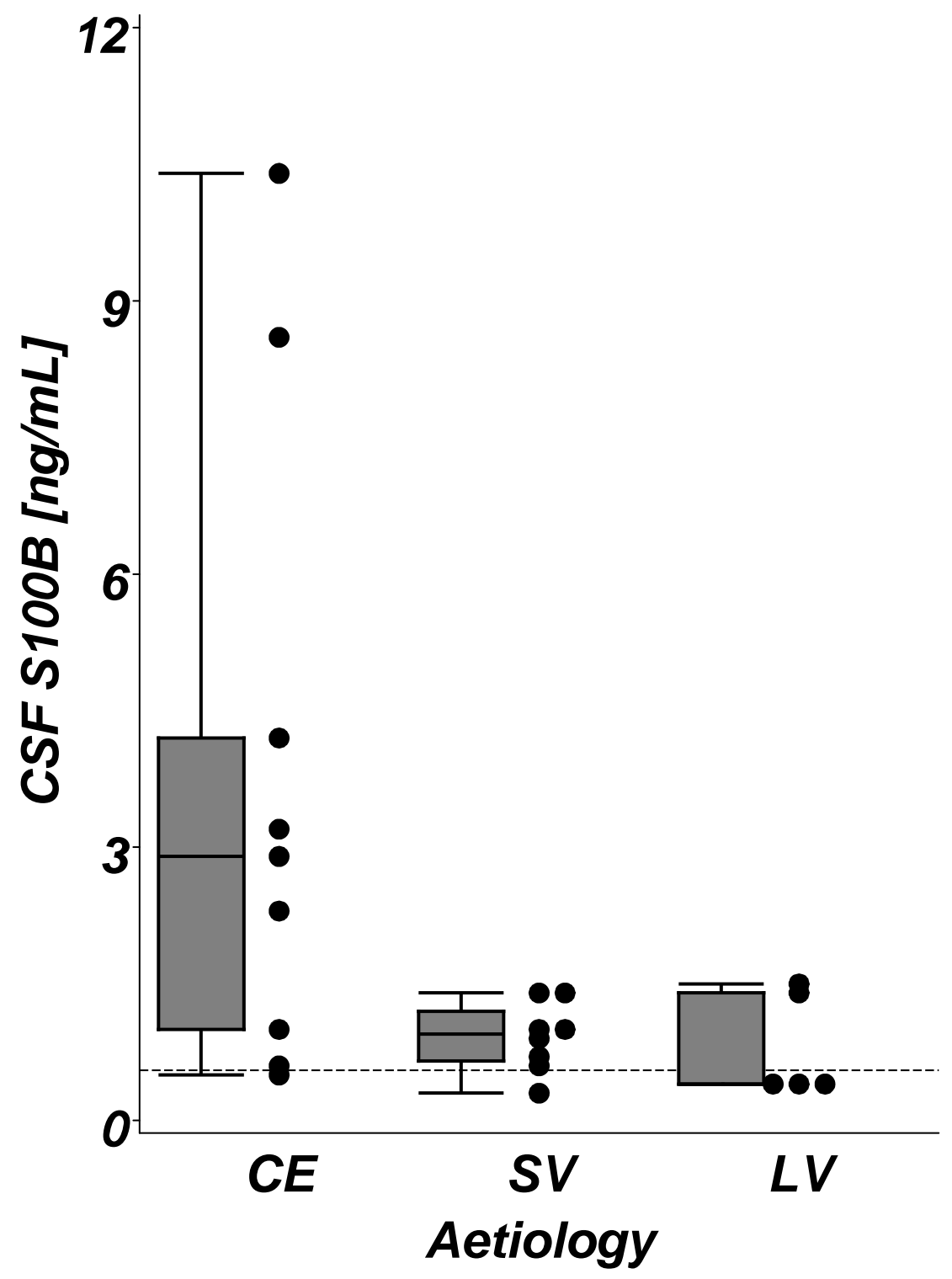

Figure 1: CSF S100B levels [ng/mL] in patients with stroke due to cardioembolic (CE), small-vessel (SV) or large-vessel (LV) aetiology. The dashed horizontal line indicates the upper reference limit of normal $(0.55$ $n g / m L) .{ }^{14}$ 
(A)

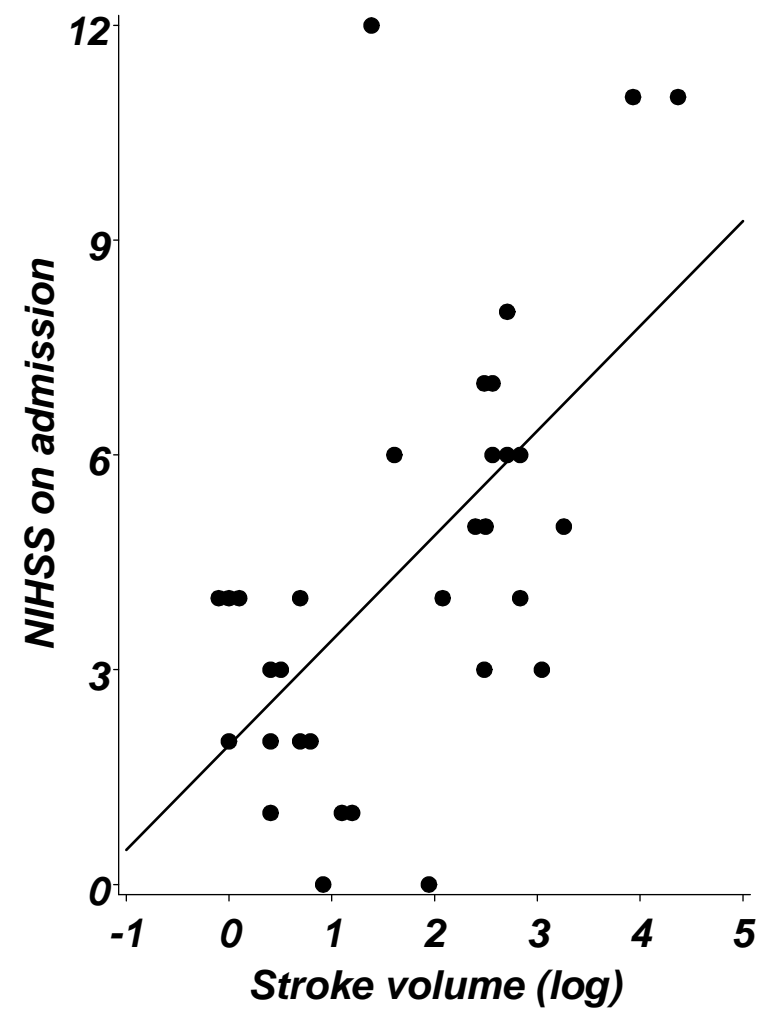

(B)

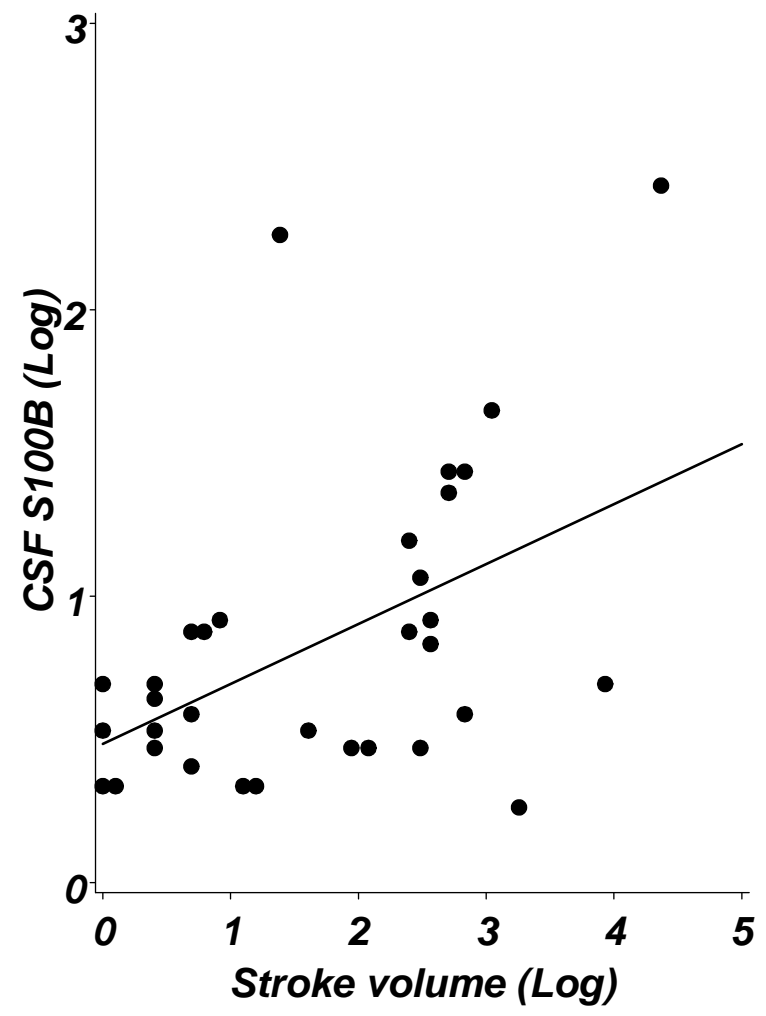

Figure 2: The stroke volume (logarithmic scale) correlated with $(A)$ the NIHSS on admission $(R=0.50, p<0.01)$ and $(B)$ the CSF S100B levels (logarithmic scale, $R=0.44, p=0.01$ ). 


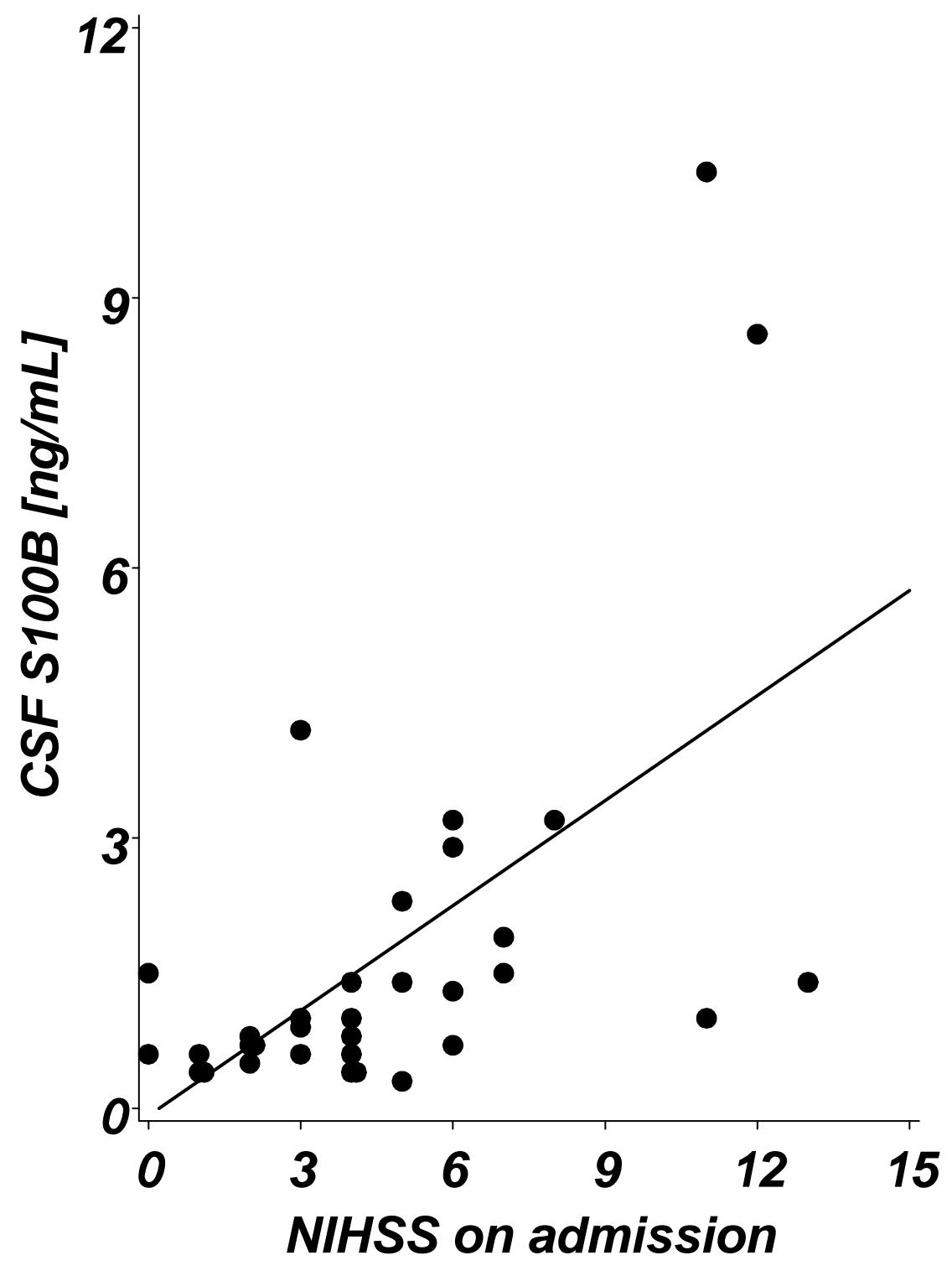

Figure 3: Stroke severity on admission (NIHSS) correlated with CSF S100B levels $(R=0.56, p<0.001)$. 